\title{
Juxtacellular Recording/Labeling Analysis of Physiological and Anatomical Characteristics of Rat Intergeniculate Leaflet Neurons
}

\author{
Stephen Thankachan ${ }^{1}$ and Benjamin Rusak ${ }^{1,2,3}$ \\ Departments of ${ }^{1}$ Psychology, ${ }^{2}$ Psychiatry, and ${ }^{3}$ Pharmacology, Dalhousie University, Halifax, Nova Scotia, Canada B3H 4J1
}

The thalamic intergeniculate leaflet (IGL) is involved in mediating effects of both photic and nonphotic stimuli on mammalian circadian rhythms. IGL neurons containing neuropeptide Y (NPY) have been implicated in mediating nonphotic effects, but little is known about those involved in photic entrainment. We used juxtacellular recording/labeling in rats to characterize both photic responses and neurochemical phenotypes of neurons in the lateral geniculate area, focusing on the IGL and ventral lateral geniculate (VLG). Single neurons were recorded to characterize photic responsiveness and were labeled with Neurobiotin (Nb); tissue was stained for Nb, NPY, and in some cases for orexin A. Three classes of neurons were identified in the IGL/VLG. Type I neurons lacked NPY and showed sustained activations during retinal illumination and moderate firing rates in darkness. Type II neurons contained large amounts of NPY throughout the soma and showed varied responses to illumination: suppression, complex responses, or no response. Type III neurons had patches of NPY both on the external soma surface and within the soma, apparently representing internalization of NPY. Type III neurons resembled type I cells in their sustained activation by illumination but were virtually silent during the intervening dark period. These neurons appear to receive NPY input, presumably from other IGL cells, which may suppress their activity during darkness. These results demonstrate the presence of several classes of neurons in the IGL defined by their functional and anatomical features and reinforce the role of the IGL/VLG complex in integrating photic and nonphotic inputs to the circadian system.

Key words: entrainment; circadian; light; intergeniculate leaflet; juxtacellular labeling; neuropeptide $Y$

\section{Introduction}

Mammalian circadian rhythms, generated by a pacemaker in the hypothalamic suprachiasmatic nucleus (SCN) (Rusak and Zucker, 1979; Meijer and Rietveld, 1989), are entrained to lighting cycles by retinal projections reaching the SCN and the thalamic intergeniculate leaflet (IGL) (Moore, 1996). Both the SCN and IGL receive binocular retinal projections (Pickard, 1985). The IGL projects to the SCN via the geniculohypothalamic tract (GHT), and IGL cells immunoreactive for neuropeptide Y (NPY) are the source of high levels of NPY in terminals in the SCN (Card and Moore, 1989; Shinohara et al., 1993; Morin and Blanchard, 2001).

Some IGL and SCN neurons respond to diffuse retinal illumination with sustained, intensity-dependent changes in firing rates (Harrington and Rusak, 1989, 1991; Meijer et al., 1989; Morin, 1994), and some photically activated IGL neurons have been shown to project to the SCN (Zhang and Rusak, 1989). Destruction of the IGL alters several effects of light on rhythms, supporting its involvement in mediating photic effects on the

\footnotetext{
Received June 29, 2005; revised Aug. 5, 2005; accepted Aug. 23, 2005

This work was supported by grants from the Canadian Institutes of Health Research (MOP67083) and Natural Sciences and Engineering Research Council of Canada (RGPIN305). We are very grateful to Debbie Fice, Donna Goguen, Stephen Whitefield, lan Meinertzhagen, Jane Anne Horne, Kazue Semba, and Satvinder Kaur for their assistance and advice.

Correspondence should be addressed to Benjamin Rusak, Department of Psychology, Life Sciences Centre, Dalhousie University, Halifax, Nova Scotia, Canada B3H 4J1. E-mail: Benjamin.Rusak@Dal.Ca. DOI:10.1523/JNEUROSCI.2672-05.2005

Copyright $\odot 2005$ Society for Neuroscience $\quad 0270-6474 / 05 / 259195-10 \$ 15.00 / 0$
}

circadian system of Syrian hamsters and rats (Harrington and Rusak, 1986; Pickard et al., 1987, 1988; Johnson et al., 1988; Edelstein and Amir, 1999).

Circadian rhythm entrainment can also be influenced by "nonphotic" factors, including increased activity and/or arousal caused by a variety of stimuli (Mrosovsky, 1996; Harrington, 1997). These effects can be quite potent and can counteract some effects of light on circadian rhythms (Biello and Mrosovsky, 1995; Schaap and Meijer, 2001). Several lines of evidence demonstrate that the IGL and its NPY projection to the SCN are involved in mediating at least some nonphotic effects on rhythms (Janik and Mrosovsky, 1992; Huhman and Albers, 1994; Edelstein and Amir, 1995; Janik et al., 1995; Biello and Mrosovsky, 1996; Marchant et al., 1997; Weber and Rea, 1997; Lall and Biello, 2003).

The IGL is, therefore, involved in both photic and nonphotic effects on the circadian system, implying heterogeneity in its cell population and connectivity. Only some IGL neurons contain NPY, colocalized with the inhibitory neurotransmitter GABA (Moore and Speh, 1993; Moore and Card, 1994). Studies of IGL function are consistent with an inhibitory input reaching the SCN from the IGL (Tang et al., 2002), mediated by GABA (Gribkoff et al., 2003), and perhaps modulated by NPY (Obrietan and van den Pol, 1996; Gribkoff et al., 1998). Other IGL neurons contain another peptide, enkephalin (Morin and Blanchard, 2001), and may not be GABAergic.

The IGL appears to convey and perhaps balance inputs from a variety of environmental cues that affect circadian rhythms. This 
function involves at least two different, but perhaps anatomically intertwined, structures represented in the IGL and in its projections to the SCN. To address the functional complexity of the IGL, we used juxtacellular labeling (Pinault, 1996). This method involves extracellular recording of the activity of a neuron followed by labeling the recorded cell selectively with Neurobiotin $\mathrm{Nb})$ to allow subsequent anatomical analyses. Using this approach, we characterized the photic responses and immunocytochemical features of neurons in the rat lateral geniculate area including the IGL.

\section{Materials and Methods}

Animals and surgery. Adult, male Sprague Dawley rats (Rattus norvegicus) from Charles River (St. Constant, Québec, Canada) weighing 250-350 g were housed in pairs under a daily $12 \mathrm{~h}$ light/dark cycle for at least $14 \mathrm{~d}$ before being used in an experiment. All experimental procedures were approved by the Dalhousie University Committee on Laboratory Animals and conformed to the guidelines of the Canadian Council on Animal Care. Rats were anesthetized with intraperitoneal injections of a $20 \%$ solution of urethane (ethyl carbonate; Sigma, St. Louis, MO) at a dose of $2 \mathrm{~g} / \mathrm{kg}$ body weight. They were also injected subcutaneously with $0.1-0.2$ $\mathrm{ml}$ of a $0.2 \%$ solution of Robinul (3-hydroxy-1,1-dimethylpyrrolidinium bromide-cyclopentylmandelate; A. H. Robins, Mississauga, Ontario, Canada), a peripheral antimuscarinic agent, to reduce congestion in the respiratory tract during anesthesia. Reflexes were checked to ensure stable anesthesia, and additional doses of urethane were given as required.

Animals were mounted in a stereotaxic apparatus with the skull level between bregma and lambda. Rectal temperature was monitored continuously and maintained at $37^{\circ} \mathrm{C}$ using a thermostatically controlled heating pad. The upper and lower eyelids of both eyes were retracted with fine sutures, and the pupils were dilated with topical application of $1 \%$ atropine sulfate. The eyes were then coated with mineral oil to prevent dehydration. Light from a tungsten-halogen lamp was infrared-filtered and led via fiber optics to both eyes. Timing of photic stimulation was programmed through a Macintosh (Apple Computers, Cupertino, CA) computer controlling an electronic shutter. The scalp was incised, overlying fascia retracted from the skull, and a small burr hole drilled over the IGL. Rats were maintained in a darkened room except during programmed light exposures or briefly while repositioning recording electrodes. Recordings were initiated during the early dark phase of the light/ dark cycle and typically were continued for $\sim 8 \mathrm{~h}$.

Neural recording and labeling. Single-unit neural activity was recorded using glass micropipettes, which were pulled to a fine tip using a PE2 electrode puller (Narishige, Tokyo, Japan) and filled with $2.5 \% \mathrm{Nb}$ (Vector Laboratories, Burlingame, CA) in $0.5 \mathrm{~m}$ sodium acetate; electrode resistance was $15-25 \mathrm{M} \Omega$. An electronic microdrive (FHC, Brunswick, ME) was used to advance the electrode to the region of the IGL (stereotaxic coordinates: $4.4-4.8 \mathrm{~mm}$ anterior to bregma, 3.9-4.1 mm lateral to midline, and $5.0-5.2 \mathrm{~mm}$ ventral to dura) (Paxinos and Watson, 1998). The electrode was positioned $\sim 1 \mathrm{~mm}$ above the IGL and then advanced in 5 $\mu \mathrm{m}$ steps until a unit with a well discriminated waveform and stable spontaneous firing was isolated. Extracellular signals were acquired using an intracellular amplifier (IR-183; Neurodata Instruments, New York, NY), discriminated using a time-amplitude window discriminator, and displayed on a storage oscilloscope. Signals were filtered ( $100 \mathrm{~Hz}$ to $2 \mathrm{kHz}$ ), fed through an analogto-digital converter (Instrutech, Port Washington, NY), and were displayed and stored to disk on a Macintosh computer using A/Dvance (written by Robert Douglas, University of British Columbia, Vancouver, British Columbia, Canada). Firing-rate histograms were generated and displayed on-line using the same program.
Once stable single-unit activity was recorded in darkness for at least 10 min, responsiveness of the cell to retinal illumination was assessed. Data were acquired at a minimum during three successive sweeps of alternating 1 min periods of darkness and binocular retinal illumination (1000 lux); longer durations of illumination were used occasionally to further assess responsiveness. Neurons were classified as photically responsive or unresponsive, as described below. After photic responsiveness was assessed, neurons were labeled using the juxtacellular labeling method described by Pinault (1996). Briefly, anodal current pulses (200 ms, 50\% duty cycle) were delivered with gradually increasing intensity $(0.5-5 \mathrm{nA})$ while continuing to record the firing activity of the neuron. After a period of stimulation ranging from a few seconds up to several minutes, the noise level of the recording increased slightly, and the neuronal activity became entrained to the pulse frequency of the applied current. The intensity of the current was then adjusted to maintain entrainment and a stable waveform without apparent damage to the cell. These current pulses both entrained the firing of the target neuron and iontophoretically ejected the $\mathrm{Nb}$ in the micropipette. Stimulation was terminated after 15-20 min, and the activity of the cell was then monitored to assess recovery of spontaneous firing activity.

Based on extensive experience with this method, the features essential to achieving excellent labeling of the target neuron with $\mathrm{Nb}$ included the following: clear discrimination of single neuronal firing with the electrode very close to the membrane (as assessed by spike amplitude); successful entrainment of neuronal firing with the positive current pulses; and successful recovery of spontaneous firing after termination of stimulation. At the end of the experiment, rats were given an overdose of urethane and were perfused transcardially with $0.9 \%$ saline $(100 \mathrm{ml})$ followed by $300 \mathrm{ml}$ of PBS containing $4 \%$ paraformaldehyde (para-PBS), $\mathrm{pH}$ 7.4. The brains were removed and postfixed in chilled $4 \%$ para-PBS overnight, followed by cryoprotection in $30 \%$ sucrose-phosphate buffer solution for $2-4 \mathrm{~d}$ at $4^{\circ} \mathrm{C}$. Coronal sections $(30 \mu \mathrm{m})$ through the recording area were cut using a cryostat, and the same sections were prepared for staining of $\mathrm{Nb}, \mathrm{NPY}$, and, in a few brains, orexin A, using conventional double- and triple-labeling immunofluorescence techniques.

Electrophysiological data analysis. Discriminated unit discharges were acquired and stored in $5 \mathrm{~s}$ bins, displayed on-line as firing-rate histograms, and stored to disk for further analysis off-line. Photically responsive neurons were identified and recorded during sustained light exposures (typically $1 \mathrm{~min}$ ) as described previously (Zhao and Rusak, 2005). Neurons were defined as photically responsive if their firing rates were consistently increased or decreased by $>30 \%$ by sustained retinal illumination. Cells that did not meet this criterion were classified as nonphotic. Cells with different photic response characteristics were often recorded very close to each other. Means of three sweeps of alternating 1 min dark and $1 \mathrm{~min}$ light periods were calculated, and average firing rates during

Table 1. Responses to retinal illumination of a sample of neurons in the lateral geniculate region

\begin{tabular}{lllll}
\hline Photically responsive & \multicolumn{3}{l}{} & \\
\hline Activated & Suppressed & Complex response & Total & \\
\hline $82(72 \%)^{a}$ & $28(25 \%)$ & $3(3 \%)$ & $113(86 \%)$ & $19(14 \%)$ \\
\hline
\end{tabular}

${ }^{a_{\text {Number }}}$ and percentage of cells recorded that met criterion for each type of photic response. Because specific response types were selectively recorded, these values do not represent the proportions of cell types in this region.

Table 2. Proportions of Neurobiotin-labeled neurons recorded in the lateral geniculate nuclei showing different responses to retinal illumination

\begin{tabular}{clllcc}
\hline & \multicolumn{2}{l}{ Photically responsive } & & Photically unresponsive & Total \\
\cline { 2 - 4 } & Activated & Suppressed & Complex response & & \\
\hline Location & & & & & \\
IGL & $25(73)^{a}$ & $4(12)$ & $2(6)$ & $3(9)$ & $34(32)$ \\
VLG & $31(74)$ & $6(14)$ & $1(2)$ & $4(10)$ & $42(40)$ \\
DLG & $14(47)$ & $12(40)$ & & $4(13)$ & $30(28)$ \\
Total & $70(66)$ & $22(21)$ & $3(3)$ & $11(10)$ & $106(100)$ \\
\hline
\end{tabular}

${ }^{a}$ Number and percentage of cells recorded that met criterion for each type of photic response. Because specific response type were selectively recorded, these values do not represent the proportions of cell types in this region. 


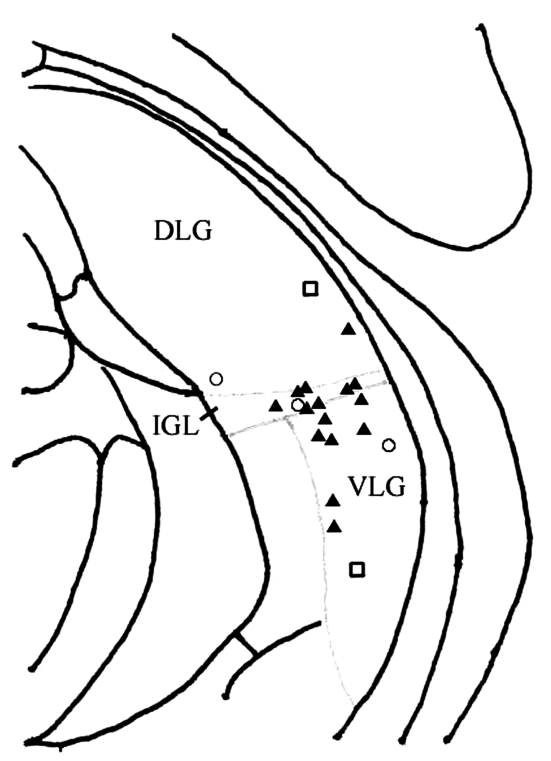

Bregma $-4.30 \mathrm{~mm}$

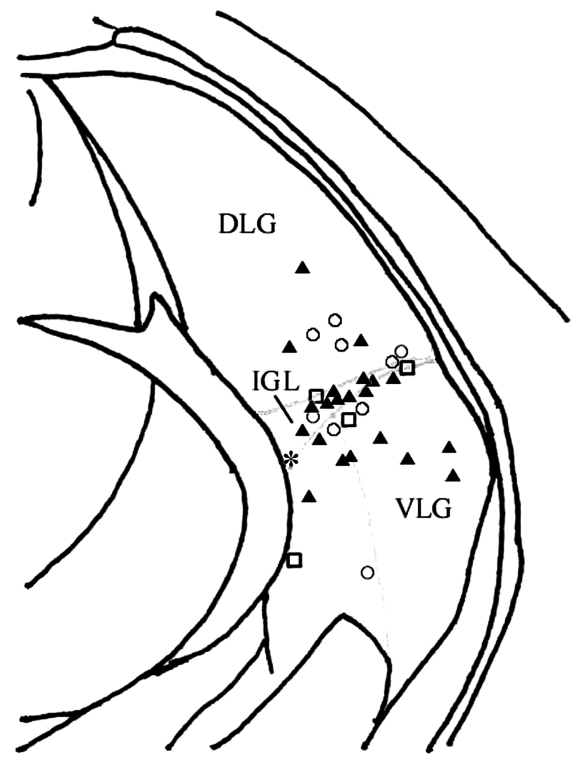

Bregma $-4.52 \mathrm{~mm}$

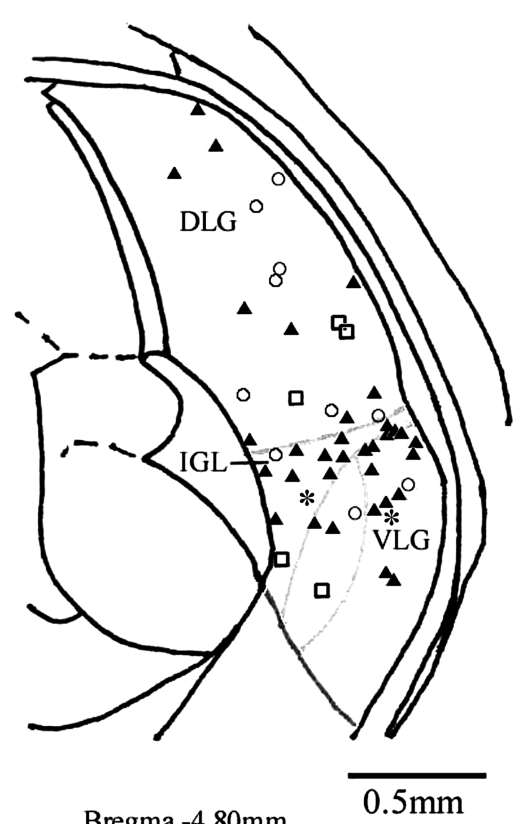

Bregma $-4.80 \mathrm{~mm}$

Figure 1. Distribution of juxtacellularly recorded neurons in the DLG nucleus, VLG nucleus, and IGL. Each symbol represents the location of a single Neurobiotin-filled neuron and its response to retinal illumination: activation $(\mathbf{\Delta})$, suppression $(\bigcirc)$, complex response $\left({ }^{*}\right)$, and lack of photic response $(\square)$. Brain sections are redrawn from the atlas of Paxinos and Watson $(1998)$.
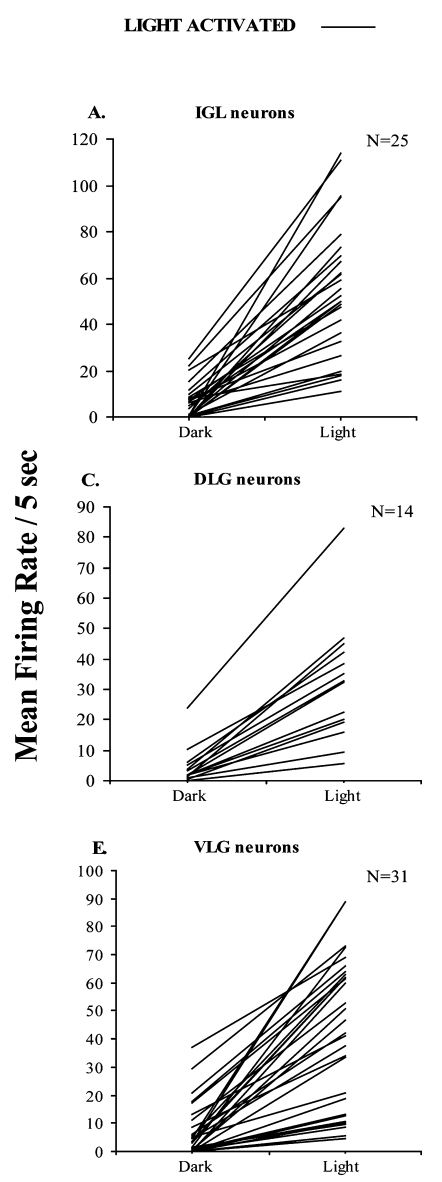

Figure 2. Average firing rates (per $5 \mathrm{~s}$ ) during baseline (Dark) and during $60 \mathrm{~s}$ light exposure (Light) of neurons in the IGL, DLG, and VLG nucleus. Neurons in $A, C$, and $E$ met criteria for activation in response to light. Those in $\boldsymbol{B}, \boldsymbol{D}$, and $\boldsymbol{F}$ were either suppressed (solid lines) or did not meet criterion for a photic response (dashed lines). Note that some DLG neurons shown as activated by light (relative to baseline) also showed transient increases at both lights-on and lights-off (see Fig. 3C).
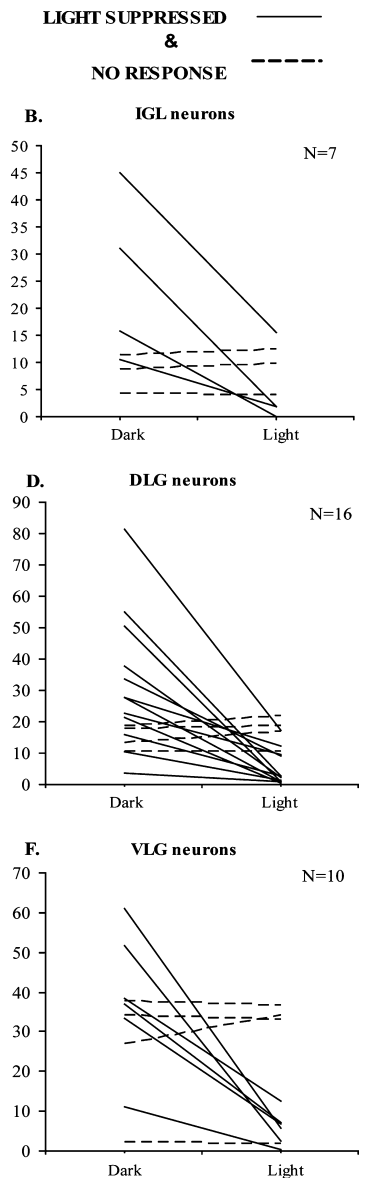

.

.
(10 and $20 \times$ objectives) were recorded by using a CCD camera and
shop to assess whether a Nb-labeled neuron was also labeled for NPY. Such double-labeled regions appeared yellow. Some neurons were also 
assessed for immunoreactivity for orexin A. Images were overlaid to identify any regions of triple labeling ( $\mathrm{Nb}, \mathrm{NPY}$, and orexin $\mathrm{A}$ ).

Confocal laser scanning microscopy was used for further analysis and to generate images at high magnification (63 and $100 \times$ objectives) that were analyzed using LSM 510 image browser software. Optical sections were taken at every $1 \mu \mathrm{m}$ depth along the $z$-axis of the $X Y$ sections. These images were stacked, and the software was used to generate $X Z$ and $Y Z$ profiles, so that labeled tissue could be visualized from three orthogonal directions simultaneously (see Figs. 5, 8). A surface-rendering program was also used to create a threedimensional (3D) image from the stack of confocal optical sections using the program Amira (v3.1.1; Mercury Computer Systems, Berlin, Germany; courtesy of Dr. I. A. Meinertzhagen, Dalhousie University, Halifax, Nova Scotia, Canada).

$\mathrm{Nb}$-labeled cells were grouped into three categories, based on degree of double-labeling. Cells that showed no overlap of NPY immunoreactivity with $\mathrm{Nb}$ labeling in the soma were classified as NPY negative. Those with high levels of NPY immunoreactivity in the central part of their somata were classified as NPYcontaining cells. A third class of cells showed small to medium patches of NPY immunoreactivity on the outside of the soma membrane, along with similar patches inside the soma, adjacent to the cell membrane. Yellow patches indicating colocalization of NPY and Nb were often limited to a single $1 \mu \mathrm{m}$ optical section in the $z$-axis or in some cases extended over two or three such sections.

\section{Results}

\section{Photic responses}

Photic responses of a total of 132 neurons in the lateral geniculate region were evaluated in detail (Table 1). Binocular illumination altered firing rates of 113 of 132 cells significantly, of which 82 of 113 $(72 \%)$ were activated, 28 of $113(25 \%)$ were suppressed, and 3 of 113 (3\%) showed complex responses. Most of these cells were recorded in the IGL, dorsal lateral geniculate (DLG), or ventral lateral geniculate (VLG) nucleus. The proportion of photically responsive cells is not representative of the population of cells in this region overall, because photically responsive cells were specifically sought for investigation.

\section{Electrophysiological and anatomical analyses of Nb-labeled neurons}

A total of 106 of the 132 neurons recorded were identified as labeled with $\mathrm{Nb}$ (green fluorescence) in the IGL, DLG, and VLG nuclei during histological analyses (Table 2, Fig. 1); Nb labeling could not be identified for the 26 remaining cells. Labeled neurons varied in size and shape. Regardless of their photic responsiveness, the majority of labeled neurons recorded in the IGL $(n=28)$ and in the VLG $(n=25)$, and a few in the DLG $(n=7)$ had small somata $(8-12 \mu \mathrm{m})$ with few dendritic fibers. A minority of cells in the IGL $(n=6)$ and many recorded in the VLG $(n=$
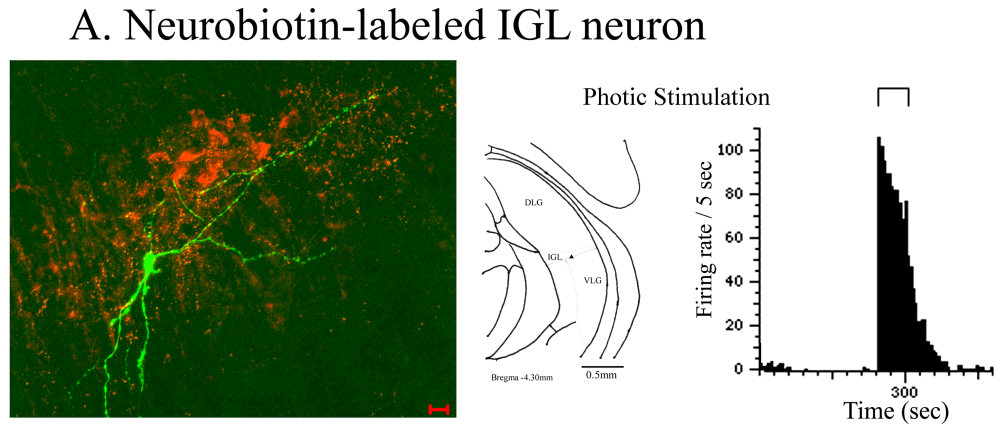

\section{B. Neurobiotin-labeled VLG neuron}
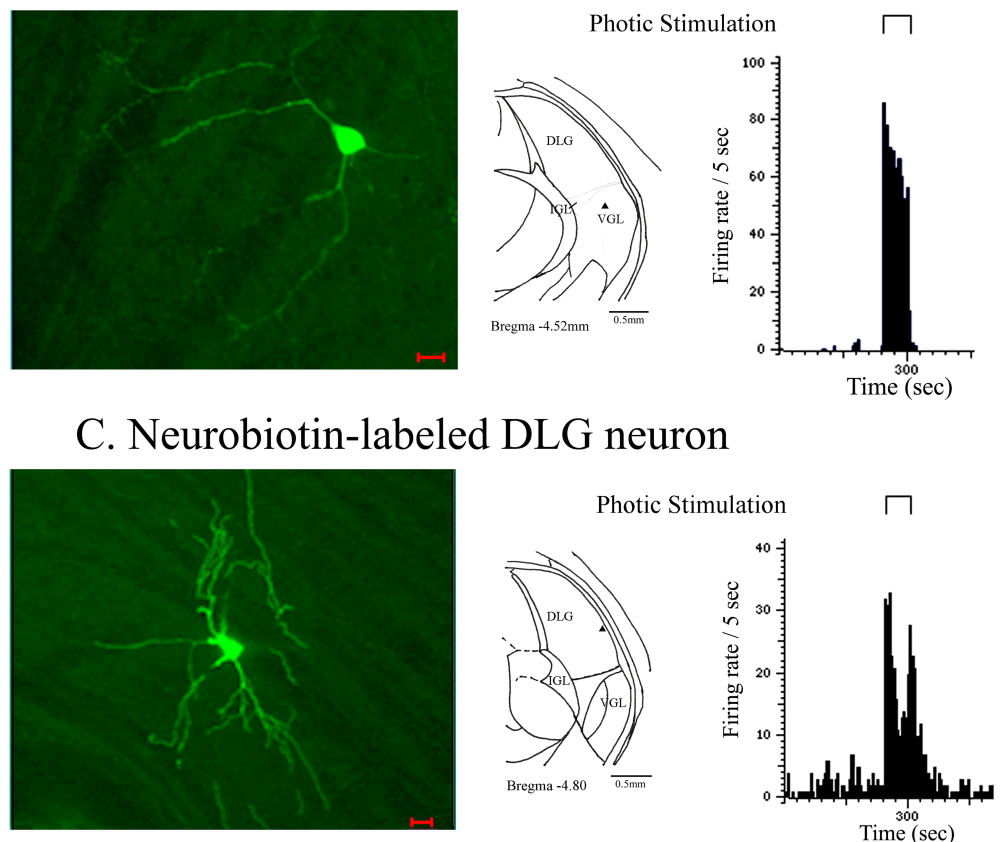

Figure 3. Examples of three juxtacellularly recorded neurons in the lateral geniculate region labeled immunocytochemically with Neurobiotin. The left column shows the Nb-labeled cell (revealed as green fluorescence by Cy2-conjugated streptavidin), and the middle column indicates its location $(\mathbf{A})$ on a schematic drawing of the region. The right column shows the baseline firing rate of the cell in aght onset, sustained high firing during illumination, and a gradual reduction to baseline in darkness. $\boldsymbol{B}, \mathrm{A}$ VLG neuron labeled as tside the NPY-immunoreactive area. It also shows sustained high firing during photic stimulation and a rapid decline to seline in darkness. C, A DLG neuron labeled as in $\boldsymbol{A}$, lying well outside the NPY-immunoreactive area. It showed prominent activations at both lights-on and lights-off, with increased firing during illumination. Scale bars, $20 \mu \mathrm{m}$ for each immunocytochemical image. Brain sections are redrawn from the atlas of Paxinos and Watson (1998).

$17)$ and especially the DLG $(n=23)$ had large somata $(15-20$ $\mu \mathrm{m})$ with extensive dendritic arborizations.

The majority of Nb-labeled neurons (70 of 106) were activated by retinal illumination, 22 were suppressed, 11 were not photically responsive (Fig. 2), and three showed complex responses that could not be readily categorized (see below). Photically activated IGL neurons (Fig. 3A) and some VLG neurons (Fig. 3B) showed a sharp increase at light onset and then a sustained elevation in firing rates at a somewhat lower level during the period of light exposure. In contrast to these cells, DLG neurons that were activated by light exposure showed more transient responses with maximal firing-rate increases in response to the onset and end of light stimulation (Fig. 3C).

Sections containing the 106 neurons labeled successfully with $\mathrm{Nb}$ were examined with fluorescence microscopy to determine the relationship of each neuron to the red, NPY-immunofluorescent cells characteristic of the IGL and to the scattered NPY-immunoflu- 
Neurobiotin-labeled IGL Neurons
A. NPY - Negative
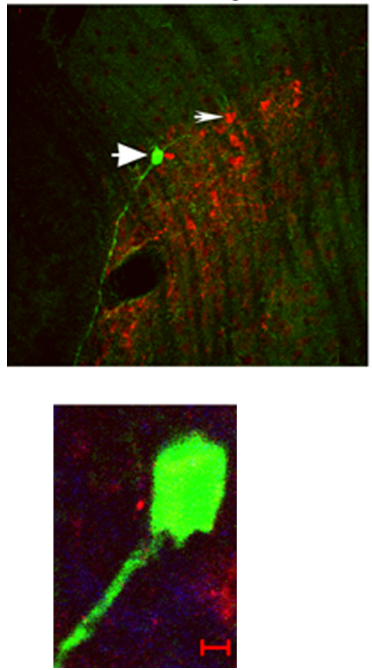

B. NPY - Negative
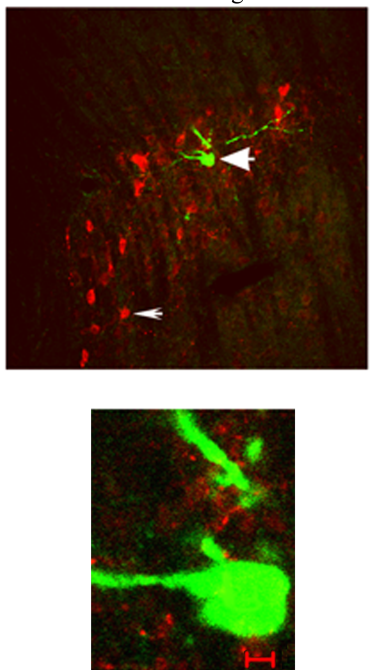

C. NPY - Positive
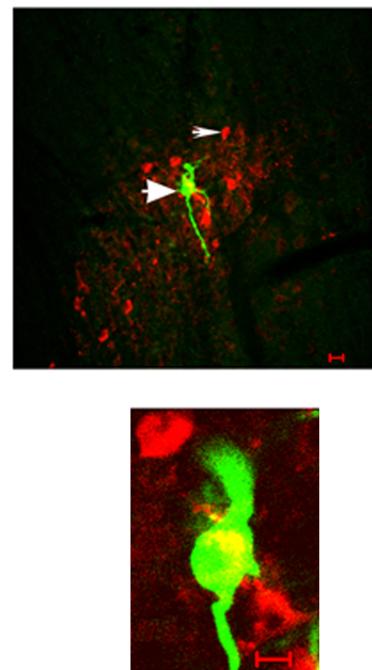

Figure 4. Top row, Confocal images showing juxtacellularly recorded, Nb-labeled neurons (green immunofluorescence, indicated by large arrows and shown as magnified images below each panel) and NPY (red immunofluorescence) marking the IGL. The scale bar in $C$ applies to all panels in the top row. Individual NPY-immunoreactive neurons are indicated by small arrows. The neurons shown in $\boldsymbol{A}$ and $\boldsymbol{B}$ were not immunoreactive for NPY. The neuron in C was immunoreactive for NPY as indicated by the double-labeling of Nb and NPY (yellow). Scale bars, $5 \mu \mathrm{m}$ for all panels.

\section{A. NPY-Negative IGL Neuron}

A1.

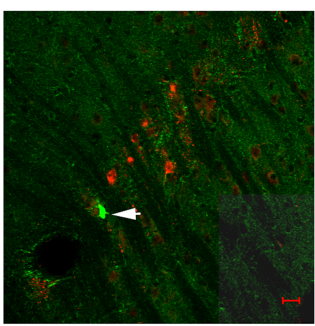

A2.

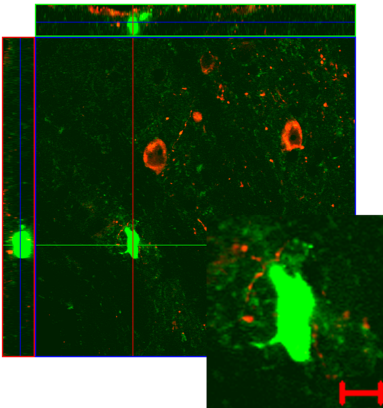

B. IGL Neuron with NPY Patches
B1.

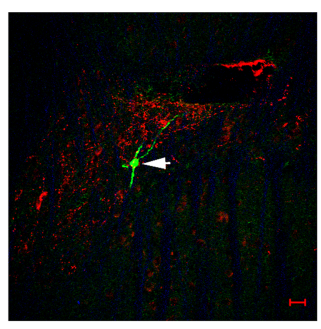

\begin{abstract}
B2. at $3 \mu \mathrm{m}$
\end{abstract}

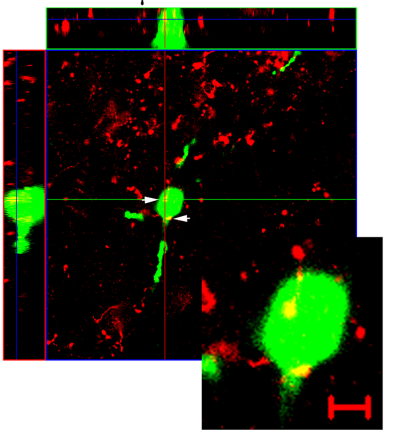

B3. at $5 \mu \mathrm{m}$

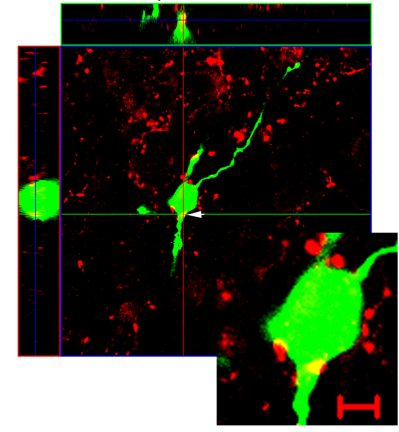

Figure 5. Confocal images of two Nb-labeled, photically activated neurons. A1, B1, The labeled neurons (green fluorescence; arrows) lie within the NPY-immunoreactive region marking the IGL (red fluorescence). A2, At higher magnification, NPYimmunoreactive somata are clearly visible adjacent to the Nb-labeled cell, which lacks any NPY immunoreactivity; Nb/NPY overlap would appear as yellow in these images. The panels above and to the left of $\mathbf{A 2}$ show the Nb-labeled soma as visualized in the two planes orthogonal to the main image. The blue line intersecting the soma in each panel indicates the level within the optical image stack from which the central image was taken. The red (vertical) and green (horizontal) lines show the levels in the central image at which orthogonal images were taken. The inset on the bottom right at very high magnification confirms the absence of NPY immunoreactivity in this neuron. $B \mathbf{B}, \boldsymbol{B}$ 3, The two larger images of the neuron in $\boldsymbol{B} 1$ were taken at different depths ( 3 and $5 \mu \mathrm{m}$ ) from the top of the optical image stack. The panels above and to the left are as described above for $\boldsymbol{A}$. These images and the very high-magnification images inset on the bottom right show patches of NPY on the external surface of the soma (red) and overlapping the soma (yellow). Scanning vertically through the entire optical image stack (191- $\mu \mathrm{m}$ optical sections) confirms that these scattered NPY patches are found both on the external surface and within the soma and extend over no more than two or three optical sections. Scale bars: $\boldsymbol{A 1}, 10$ $\mu \mathrm{m} ; \boldsymbol{B} 1,20 \mu \mathrm{m} ; A \mathbf{2}, \mathbf{B} 2, \boldsymbol{B} 3,5 \mu \mathrm{m}$. orescent cells in the VLG. The 30 cells recorded in the DLG included all response types, and, as would be predicted by the general lack of NPY in the DLG, none were NPY positive. Of the $76 \mathrm{Nb}$-labeled neurons recorded in the IGL or VLG, 56 were activated, 10 were suppressed, seven were unresponsive (Fig. $2 A, B, E, F$ ), and three (two in IGL and one in VLG) showed complex responses. One of these, at least, appeared to be primarily suppressed by the onset of illumination, but the response was variable and followed by apparent rebound activation (see Fig. 7C).

Of the 76 IGL/VLG neurons, 57 showed no NPY immunofluorescence; these included activated (44), suppressed (eight), and unresponsive (five) neurons. Among these cells, 13 (all activated) lay within, or immediately adjacent to, the dense band of NPY-immunofluorescent cells marking the IGL. These NPY-negative IGL neurons and 19 IGL/VLG neurons that showed some degree of NPY immunofluorescence were studied further using confocal laser scanning microscopy. Representative confocal images illustrating the location of three $\mathrm{Nb}$-immunofluorescent neurons (green) within the IGL show two that do not contain NPY (Fig. 4A,B) but are adjacent to NPY-immunofluorescent neurons (red) and one that does contain NPY (yellow, indicating Nb and NPY colocalization) (Fig. 4C).

Based on their NPY immunofluorescence, the 32 neurons studied with confocal microscopy were divided into three groups. Type I Nb-labeled neurons in the IGL region lacked NPY labeling $(n=13)$ (Fig. $5 A$ ). They were activated sharply by light onset and showed sustained firing during light exposure and a rapid decline of firing rate after return to darkness. These cells also had a low-to-moderate firing rate during darkness (Fig. $6 A$ ).

Type II neurons, comprising seven of the $32 \mathrm{Nb}$-labeled neurons in the IGL/ VLG region, had somata containing substantial NPY immunofluorescence that was visible in all three planes of optical section. These neurons were suppressed by light (two neurons), unresponsive (two neurons), or showed complex responses dominated by initial suppression and with evidence of activation at lights-off (three neurons). None of the seven NPY-positive neurons were activated by onset of retinal illumination, as the 13 NPY-negative neurons were. One NPY-immunofluorescent, Nb-labeled neuron that showed a complex response to retinal illumination is illustrated in Figures 7 and 8. A 3D reconstruction created using Amira software 

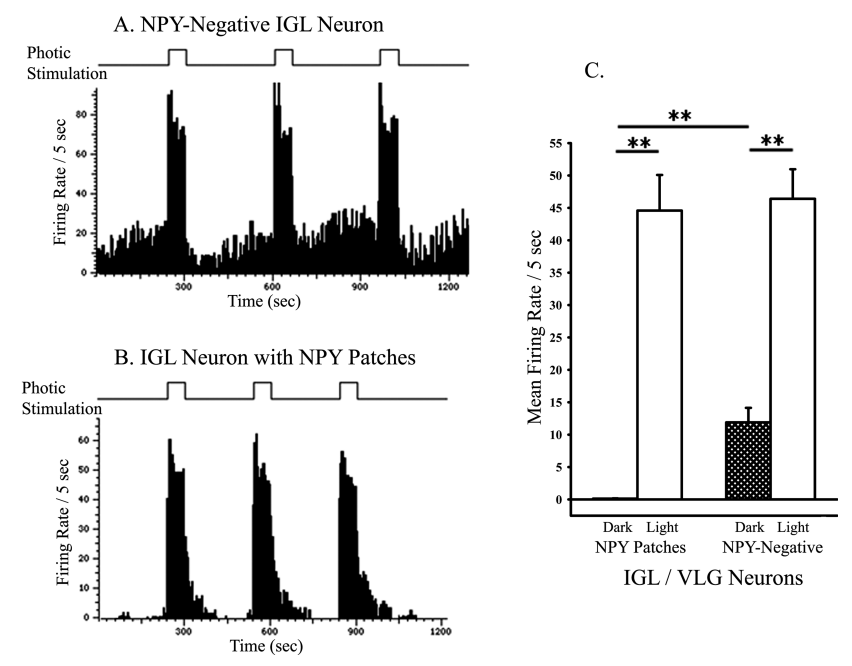

Figure 6. A, Firing-rate histogram of responses to retinal illumination by the NPY-negative, $\mathrm{Nb}$-labeled IGL neuron illustrated in Figure $5 A$. The cell showed a moderate firing rate in darkness, a sharp increase in firing at illumination onset (upward deflections of the line above the histogram), and sustained high firing during each 60 s light exposure. $\boldsymbol{B}$, Firing-rate histogram of response to retinal illumination by the Nb-labeled IGL cell illustrated in Figure $5 B$, with patches of NPY immunoreactivity within and on the surface of the soma. This neuron was virtually silent in darkness, showed a sharp increase in firing at illumination onset, sustained firing during 60 s illumination (upward deflection), and showed a gradual decline to baseline at lights-off. C, A histogram showing the mean baseline firing rates in the dark and during $60 \mathrm{~s}$ light exposures for light-activated IGL/VLG neurons with NPY patches in and on the soma (left; $n=12$ ) and with no NPY immunoreactivity (right; $n=13$ ). There was a statistically significant increase in firing rate from dark to light in both groups and a statistically significant difference in baseline firing rate in the dark between the groups. Average firing rates during light exposure were virtually identical for these two groups $\left({ }^{* *} p<0.01\right)$. Error bars represent SEM.

illustrates the pattern of immunofluorescence for NPY within this neuron (Fig. 8). These images illustrate the characteristics these cells had in common: substantial NPY content in the core of the soma and a lack of substantial NPY immunoreactivity directly on the soma surface.

Type III neurons comprised the 12 remaining Nb-labeled neurons in the IGL/VLG region. They differed from the other types in that they showed distinctive patches of NPY immunoreactivity on their soma surfaces in standard confocal images (Fig. $5 B$ ). Reconstructed 3D images of these neurons revealed that there were also small patches of NPY immunofluorescence associated with the inside walls of their somata. In many cases, these patches appeared to be continuous with patches on the external surface of the somata (Fig. 9). Unlike the type II cells described above as NPY positive, these neurons did not contain a large region of dense NPY immunofluorescence in the core of their somata.

We interpreted the external patches as representing clusters of NPY-containing terminals reaching these neurons and the internal patches as representing internalization of NPY peptide/receptor complexes, as observed with other peptides (Faure et al., 1995; Csaba et al., 2001; Morinville et al., 2004). To help evaluate this interpretation, we examined immunoreactivity for another peptide, orexin A, within the IGL (Fig. 10A). Orexin A reaches the IGL from cells in the lateral hypothalamus (Peyron et al., 1998) but is not produced within IGL neurons. Orexin A-immunofluorescent patches were detected on the surface and within the soma of an IGL neuron (Fig. 10 B2); these appeared to be morphologically similar to the NPY patches seen in type III neurons. This same neuron was identified as NPY-containing (type II), based on the characteristic dense NPY immunofluorescence throughout its soma (Fig. 10 B1). As did some other NPY-containing neurons, this cell showed a complex response to retinal illumination characterized by suppression of firing at light onset.

All type III neurons (eight in IGL and four in VLG) showed sharp activations in response to the onset of retinal illumination, sustained activation during illumination, and a gradual decline to baseline firing in darkness. A striking difference between these cells showing NPY patches and the type I neurons with similar activations in response to light that lacked such patches (see above) was the virtual silence of type III neurons during darkness (Fig. 6B, $C$ ). These neurons, interpreted as receiving NPY projections, had a mean firing rate in darkness of $0.1 \pm 0.04$ spikes per $5 \mathrm{~s}$, whereas type I neurons that lacked NPY patches on their somata had mean firing rates in darkness of $11.9 \pm 2.2$ spikes per $5 \mathrm{~s}(p<0.01$ by $t$ test). In contrast to these observations, type I and III neurons showed very similar firing rates during retinal illumination (type I, $44.6 \pm 5.5$ spikes per 5 s; type III, $46.0 \pm 4.6$ spikes per $5 \mathrm{~s}$; NS) (Fig. 6C).

\section{Discussion}

The use of the juxtacellular recording/labeling method revealed three classes of neurons in the IGL and adjacent VLG, distinguished by their NPY content and their electrophysiological responses to illumination conditions. Based on previous anatomical studies (Mantyh and Kemp, 1983; Takatsuji and Tohyama, 1989; Moore and Card, 1994), we anticipated the presence of neurons that did and did not contain NPY, but we also identified a third class of cells that contained small amounts of NPY within the soma, associated with patches of NPY on the soma surface. The patchy distribution of NPY both within and outside the soma, and the similarity of this pattern to that observed for orexin A, which is found in the IGL only in afferent fibers, suggest that these cells receive NPY afferents and internalize NPY peptide/ receptor complexes. Similar patterns of peptide/receptor internalization have been reported previously for neurotensin and for somatostatin receptors in neurons (Faure et al., 1995; Csaba et al., 2001; Morinville et al., 2004) and for NPY and its $\mathrm{Y}_{1}$ receptor in mammalian cell lines (Gicquiaux et al., 2002; Pheng et al., 2003).

These three types of cells responded differently to retinal illumination. Type I neurons lacking NPY showed sharp increases in firing at light onset, sustained activations during continued illumination, and moderate firing rates in darkness. Some IGL cells with similar responses to illumination have been shown to project to the SCN (Zhang and Rusak, 1989). It is likely that light-activated IGL/VLG neurons that lack NPY are involved in mediating effects of light on phase shifting and on the freerunning period of circadian rhythms generated by the SCN (Harrington and Rusak, 1986, 1988; Pickard et al., 1987).

In contrast, type II neurons that contain large amounts of NPY throughout the soma were not similarly activated by illumination. These cells also likely contribute to the GHT projection to the SCN (and pretectum), because NPY-containing cells form part of these projections (Harrington et al., 1985, 1987; Card and Moore, 1989; Morin and Blanchard, 2001). Type II cells showed heterogeneous responses to illumination, including sustained suppression, transient suppression with rebound activation (either during or after light exposure), or a lack of photic responsiveness. The complex and sometimes variable responses to illumination suggest that these neurons may receive both direct retinal projections and indirect input from other regions, such as the contralateral IGL or local interneurons (Card and Moore, 1989; Zhang and Rusak, 1989; Vrang et al., 2003). Because NPYimmunoreactive cells respond to nonphotic stimuli with increased c-Fos expression (Janik and Mrosovsky, 1992), and be- 
A. Neuron Location
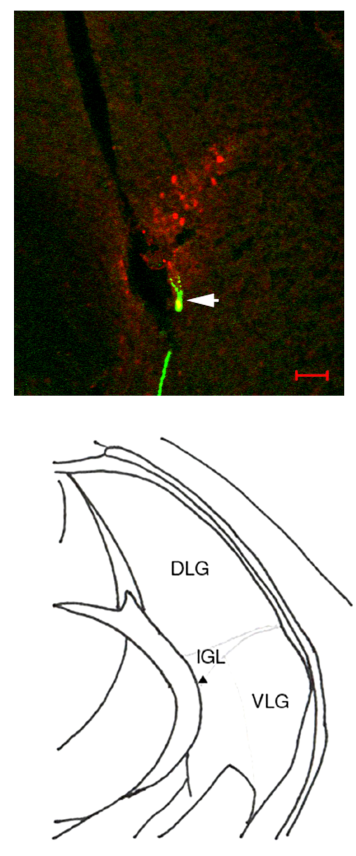

Bregma $-4.52 \mathrm{~mm} \overline{0.5 \mathrm{~mm}}$

Photic

Stimulation
B1. Neurobiotin

B2. Neuropeptide Y

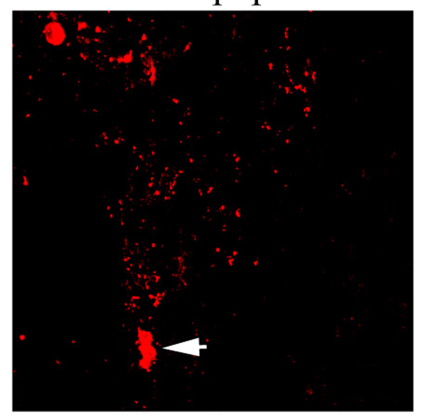

C. Photic Responses

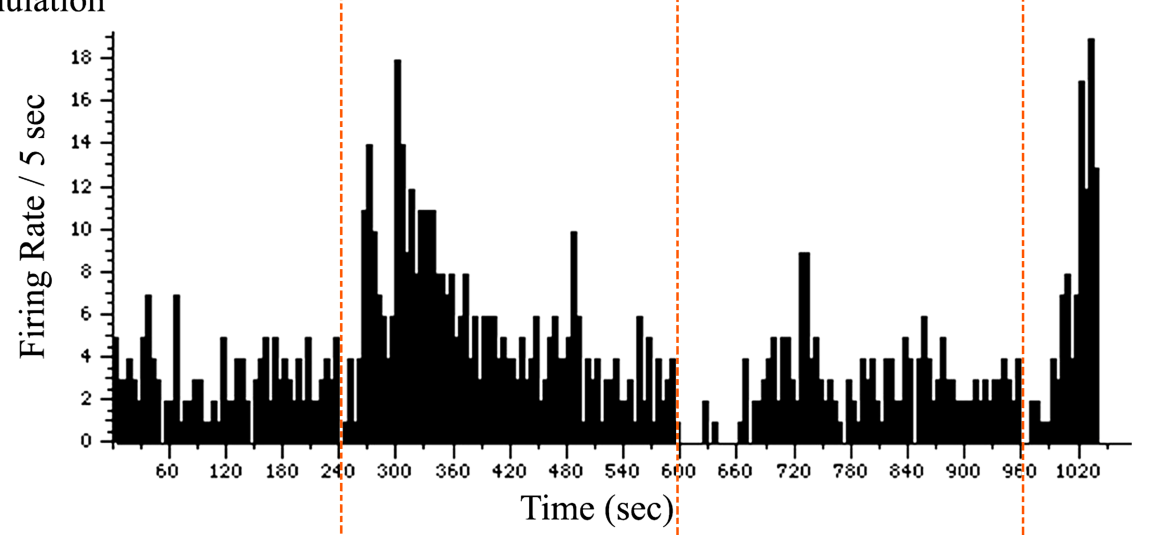

Figure 7. Anatomical and neurophysiological characteristics of a photically responsive neuron in the IGL. The arrow in each panel points to the same neuronal soma. $\boldsymbol{A}$, A low-power image of the region of the IGL showing the location of the neuron (double-labeled) at the ventromedial edge of the IGL. The bottom panel shows a schematic of the area indicating the position of the neuron ( $\mathbf{A}$ ). $\boldsymbol{B} 1$, Green fluorescence labeling indicating the presence of $\mathrm{Nb}$ in the cell soma after juxtacellular labeling. $\mathbf{B 2}$, Red fluorescence labeling showing NPY immunoreactivity in the IGL and in the soma of this neuron. $\boldsymbol{B 3}$, An overlapped image of $\boldsymbol{A}$ and $\boldsymbol{B}$ showing that this Nb-labeled neuron is immunoreactive for NPY. Yellow indicates overlap of Nb and NPY. $\boldsymbol{C}$, Firing-rate histogram illustrating responses of this neuron to binocular retinal illumination (60 s), indicated by the upward deflection of the line above the histogram and by a vertical dashed line. The complex responses include an initial suppression of firing at lights-on in all three repetitions. In the first and third examples, this is followed by a rebound activation during illumination and increased firing at lights-off. The second example shows only strong suppression to illumination. Scale bars: $\boldsymbol{A}, 50 \mu \mathrm{m}$; (in B3) $\boldsymbol{B} 1-\boldsymbol{B} 3,20 \mu \mathrm{m}$. The brain section is redrawn from the atlas of Paxinos and Watson (1998).

cause NPY release in the SCN appears to be essential for phase shifts induced by arousal and similar nonphotic stimuli (Marchant et al., 1997; Maywood et al., 1997), type II cells are likely candidates for mediating these nonphotic effects (Harrington, 1997). The lack of sustained activations in response to illumination in NPY-containing type II cells and the lack of NPY in photically activated type I cells are consistent with the reported mutual antagonism of the effects of light and of nonphotic stimuli on circadian phase (Biello and Mrosovsky, 1995; Biello et al., 1997).

It is unclear what the net responses of type II cells showing initial suppressions and rebound activations would be to longer light exposures, such as those used in studies of light-evoked gene expression in the IGL (Park et al., 1993; Edelstein and Amir, 1996). Increased c-Fos expression in the IGL after light exposure differs from that in most SCN cells (Rusak et al., 1990; Abe and Rusak, 1994) in that it does not depend on circadian phase (Park et al., 1993; Edelstein et al., 2000) and is not attenuated by previous blockade of glutamatergic transmission (Edelstein and Amir, 1998). c-Fos expression in IGL cells may be modulated by direct retinal input, via interneurons, and in vivo by the behavioral consequences of light exposure.

Type III cells resembled type I cells in showing robust, sustained activations in response to retinal illumination but differed in being virtually silent during intervening dark periods. This profound suppression during darkness correlated with the pres- ence of NPY patches both within the soma and on its external surface. Our interpretation of these observations is that these cells receive NPY-containing projections, possibly from other IGL neurons. Type II neurons that contain NPY may be suppressed by light and activated by darkness (see above); their increased firing and release of NPY during darkness may suppress firing in NPYrecipient type III IGL neurons to which they project, thereby accounting for the virtual silence of type III neurons in darkness. Electrical stimulation of the contralateral IGL in hamsters suppresses photic responses of IGL cells (Zhang and Rusak, 1989), but anatomical evidence in rats and hamsters (Card and Moore, 1989; Morin and Blanchard, 1995) suggests that NPY is not a major part of the projection from the contralateral IGL. The arborizations of type II neurons within the IGL (Figs. 4C, 7) provide a likely substrate for ipsilateral NPY input to other IGL neurons.

Internalization of NPY-receptor complexes into neurons in the brain has not been described previously. There are, however, several reports of ligand-stimulated internalization of both NPY and its $\mathrm{Y}_{1}$ receptors in mammalian cell lines (Gicquiaux et al., 2002; Pheng et al., 2003) and for similar internalization of other peptides into neurons in vivo (Faure et al., 1995; Csaba et al., 2001; Morinville et al., 2004). Our interpretation of the staining pattern in type III neurons is also supported by several other lines of evidence. Many internal NPY patches were apparently continuous with similar patches on the external soma surface (Fig. 9). 


\section{Neurobiotin and NPY}

\section{A. Confocal Image}

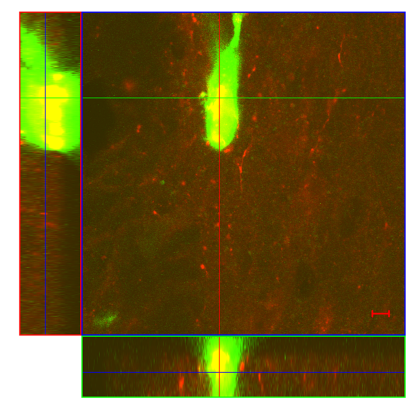

B. $3 \mathrm{D}$

\section{Reconstruction}

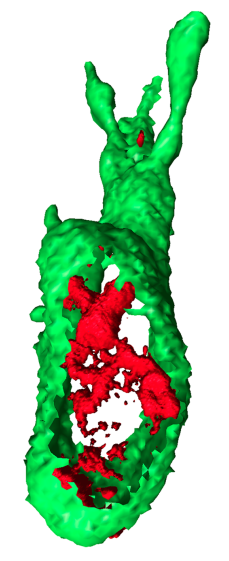

\section{Rotated 3D Reconstruction}

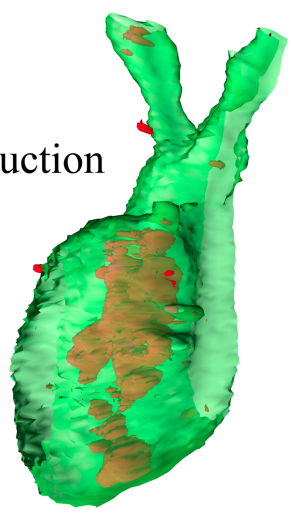

Figure 8. Confocal images and $3 D$ reconstructions of the NPY-containing IGL neuron documented in Figure 7. A, High-magnification confocal images showing immunoreactivity for $\mathrm{Nb}$ (green) and NPY (red) and their overlap (yellow). Scale bar, $5 \mu \mathrm{m}$. B, A 3D reconstruction (using Amira software) of the entire stack of 19 optical sections with the soma "opened up" from the viewer's perspective, showing the extensive NPY content (red) of the neuron. C, A 3D surfacerendered reconstruction that is rotated relative to $\boldsymbol{B}$ and with the soma wall smoothed and made partially transparent to allow visualization of its antigen content.

Cells without internal NPY patches also lacked NPY patches on the external soma surface, and these cells showed moderate neural activity in darkness, suggesting a lack of NPY-mediated suppression. In addition, there was a similar pattern of staining in some IGL neurons for orexin A, which originates from projections to IGL neurons (Peyron et al., 1998).

It is unclear whether the degree of apparent NPY internalization in type III neurons relates to the patterns of light exposure animals experienced during the study. Repeated exposure to alternating light and dark periods may have promoted activation of NPY-ergic neurons and the release, binding, and subsequent in- ternalization of NPY into target neurons. But it is also possible that this process occurs independently of changes in photic stimulation or could be affected by anesthesia.

All neurons with NPY patches were activated by light, whereas those with NPY-filled somata were not, supporting the interpretation that these represent different classes of neurons. The available data, however, cannot provide definitive evidence on this issue. In principle, an NPY-receptive cell might contain sufficient internalized NPY to fill most of the soma. Future studies should combine juxtacellular labeling with immunocytochemical and in situ hybridization methods to confirm the functional characteristics of IGL cells that contain mRNAs for NPY or for its receptors (cf. Stornetta et al., 1999).

These data support the idea of two functionally separate but anatomically interwoven IGL systems that are involved in photic and nonphotic entrainment processes (cf., Card and Moore, 1989; Abe and Rusak, 1992). IGL neurons that respond to retinal illumination with activation do not produce NPY and are likely candidates for mediating the effects of IGL ablation on photic responsiveness of the circadian system (Harrington and Rusak, 1986; Pickard et al., 1987, 1988; Edelstein and Amir, 1999). These photically activated neurons, however, appear to be comprised of two populations: those that do and do not receive NPY projections. The functional significance of these two classes of IGL neurons is unknown. Also unknown are the cell types that contribute to the widespread IGL connections with other brain regions and contribute to possible noncircadian functions of this nucleus (Mikkelsen, 1990; Moga and Moore, 1997; Vrang et al., 2003; Morin and Blanchard, 2005).

Neurons that contain large amounts of NPY (putatively NPYproducing) did not respond to increased illumination with activation. These cells are likely responsible for the role of the IGL in mediating the phase-shifting effects of various nonphotic cues that are mediated by NPY (Harrington, 1997). The heterogeneous responses of these NPY-containing cells included suppression by light (activation by dark), which is consistent with the reported antagonism between the effects of NPY and of light on the circadian system (Biello and Mrosovsky, 1995; Biello et al., 1997). Photically unresponsive cells that contain NPY may respond to other inputs related to nonphotic phase shifting, such as serotonergic input from the raphe nuclei (Meyer-Bernstein and Morin, 1996; Marchant et al., 1997).

In summary, these studies have characterized the immunocytochemical characteristics of IGL/VLG neurons that respond differently to retinal illumination. The results indicate that there are several anatomical and functional classes of neurons in this region that may be involved in mediating different kinds of inputs to the circadian system. These findings reinforce the role of the IGL/VLG complex in integrating photic and nonphotic inputs to modulate circadian rhythm entrainment.

\section{References}

Abe H, Rusak B (1992) Stimulation of the hamster ventral lateral geniculate nucleus induces Fos-like immunoreactivity in suprachiasmatic nucleus cells. Neurosci Lett 148:185-189.

Abe H, Rusak B (1994) Physiological mechanisms regulating photic induction of Fos-like protein in hamster suprachiasmatic nucleus. Neurosci Biobehav Rev 18:531-536.

Biello SM, Mrosovsky N (1995) Blocking the phase-shifting effect of neuropeptide Y with light. Proc R Soc Lond B Biol Sci 259:179-187.

Biello SM, Mrosovsky N (1996) Phase response curves to neuropeptide Y in wild type and tau mutant hamsters. J Biol Rhythms 11:27-34.

Biello SM, Golombek DA, Harrington ME (1997) Neuropeptide Y and glutamate block each other's phase shifts in the suprachiasmatic nucleus in vitro. Neuroscience 77:1049-1057. 


\section{IGL Neuron with NPY Patches}

A.

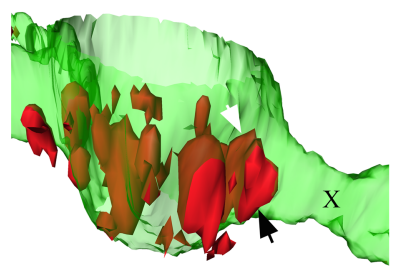

B.

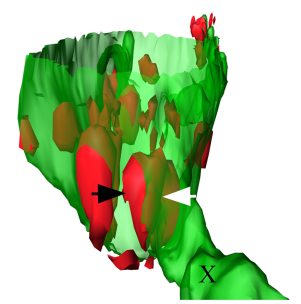

C.

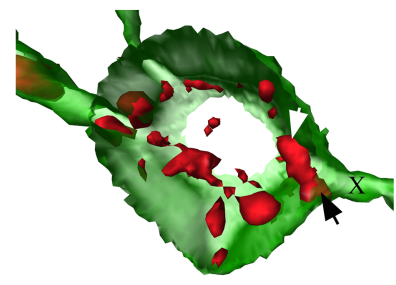

Figure 9. Surface-rendered views of the soma of a light-activated IGL neuron, labeled with Nb (green) and with patches of NPY (red), both on the external surface and within the walls of the soma (made partially transparent). Each panel shows the same neurite marked with an $X$ as a position reference. The images have been rotated freehand to show the patches from different perspectives. In each panel, the black arrow indicates the same patch of NPY immunoreactivity on the external soma surface, and the white arrow indicates the NPY within the soma continuous with that patch.

Card JP, Moore RY (1989) Organization of lateral geniculate-hypothalamic connections in the rat. J Comp Neurol 284:135-147.

Csaba Z, Bernard V, Helboe L, Bluet-Pajot M-T, Bloch B, Epelbaum J, Dournaud $P$ (2001) In vivo internalization of the somatostatin sst2A receptor in rat brain: evidence for translocation of cell-surface receptors into the endosomal recycling pathway. Mol Cell Neurosci 17:646-661.

Edelstein K, Amir S (1995) Non-photic manipulations induce expression of Fos protein in the suprachiasmatic nucleus and intergeniculate leaflet in the rat. Brain Res 690:254-258.

Edelstein K, Amir S (1996) Constant light induces persistent Fos expression in rat intergeniculate leaflet. Brain Res 731:221-225.

Edelstein K, Amir S (1998) Glutamatergic antagonists do not attenuate lightinduced FOS protein in rat intergeniculate leaflet. Brain Res 810:264-268.

Edelstein K, Amir S (1999) The role of the intergeniculate leaflet in entrainment of circadian rhythms to a skeleton photoperiod. J Neurosci 19:372-380.

Edelstein K, Beaule C, D’Abramo R, Amir S (2000) Expression profiles of JunB and c-Fos proteins in the rat circadian system. Brain Res 870:54-65.

Faure MP, Alonso A, Nouel D, Gaudriault G, Dennis M, Vincent JP, Beaudet A (1995) Somatodendritic internalization and perinuclear targeting of neurotensin in the mammalian brain. J Neurosci 15:4140-4147.

Gicquiaux H, Lecat S, Gaire M, Dieterlen A, Mely Y, Takeda K, Bucher B, Galzi JL (2002) Rapid internalization and recycling of the human neuropeptide Y Y(1) receptor. J Biol Chem 277:6645-6655.

Gribkoff VK, Pieschl RL, Wisialowski TA, van den Pol AN, Yocca FD (1998) Phase shifting of circadian rhythms and depression of neuronal activity in the rat suprachiasmatic nucleus by neuropeptide Y: mediation by different receptor subtypes. J Neurosci 18:3014-3022.

Gribkoff VK, Pieschl RL, Dudek FE (2003) GABA receptor-mediated inhibition of neuronal activity in rat SCN in vitro: pharmacology and influence of circadian phase. J Neurophysiol 90:1438-1448.

Harrington ME (1997) The ventral lateral geniculate nucleus and the intergeniculate leaflet: interrelated structures in the visual and circadian systems. Neurosci Biobehav Rev 21:705-727.

Harrington ME, Rusak B (1986) Lesions of the thalamic intergeniculate leaflet alter hamster circadian rhythms. J Biol Rhythms 1:309-325.

Harrington ME, Rusak B (1988) Ablation of the geniculo-hypothalamic tract alters circadian activity rhythms of hamsters housed under constant light. Physiol Behav 42:183-189.

Harrington ME, Rusak B (1989) Photic responses of geniculo-hypothalamic tract neurons in the Syrian hamster. Vis Neurosci 2:367-375.

Harrington ME, Rusak B (1991) Luminance coding properties of intergeniculate leaflet neurons in the golden hamster and the effects of chronic clorgyline. Brain Res 554:95-104.

Harrington ME, Nance DM, Rusak B (1985) Neuropeptide Y immunoreactivity in the hamster geniculo-suprachiasmatic tract. Brain Res Bull 15:465-472.

Harrington ME, Nance DM, Rusak B (1987) Double-labeling of neuropeptide $\mathrm{Y}$-immunoreactive neurons which project from the geniculate to the suprachiasmatic nuclei. Brain Res 410:275-282.

Huhman KL, Albers HE (1994) Neuropeptide Y microinjected into the su- prachiasmatic region phase shifts circadian rhythms in constant darkness. Peptides 15:1475-1478.

Janik D, Mrosovsky N (1992) Gene expression in the geniculate induced by a nonphotic circadian phase shifting stimulus. NeuroReport 3:575-578.

Janik D, Mikkelsen JD, Mrosovsky N (1995) Cellular colocalization of Fos and neuropeptide $\mathrm{Y}$ in the intergeniculate leaflet after nonphotic phase-shifting events. Brain Res 698:137-145.

Johnson RF, Smale L, Moore RY, Morin LP (1988) Lateral geniculate lesions block circadian phase-shift responses to a benzodiazepine. Proc Natl Acad Sci USA 85:5301-5304.

Lall GS, Biello SM (2003) Attenuation of circadian light induced phase advances and delays by neuropeptide $\mathrm{Y}$ and a neuropeptide $\mathrm{Y}$ Y1/Y5 receptor agonist. Neuroscience 119:611-618.

Mantyh PW, Kemp JA (1983) The distribution of putative neurotransmitters in the lateral geniculate nucleus of the rat. Brain Res 288:344-348.

Marchant EG, Watson NV, Mistlberger RE (1997) Both neuropeptide Y and serotonin are necessary for entrainment of circadian rhythms in mice by daily treadmill running schedules. J Neurosci 17:7974-7987.

Maywood ES, Smith E, Hall SJ, Hastings MH (1997) A thalamic contribution to arousal-induced, non-photic entrainment of the circadian clock of the Syrian hamster. Eur J Neurosci 9:1739-1747.

Meijer JH, Rietveld WJ (1989) Neurophysiology of the suprachiasmatic circadian pacemaker in rodents. Physiol Rev 69:671-707.

Meijer JH, Rusak B, Harrington ME (1989) Photically responsive neurons in the hypothalamus of a diurnal ground squirrel. Brain Res 501:315-323.

Meyer-Bernstein EL, Morin LP (1996) Differential serotonergic innervation of the suprachiasmatic nucleus and the intergeniculate leaflet and its role in circadian rhythm modulation. J Neurosci 16:2097-2111.

Mikkelsen JD (1990) Projections from the lateral geniculate nucleus to the hypothalamus of the Mongolian gerbil (Meriones unguiculatus): an anterograde and retrograde tracing study. J Comp Neurol 299:493-508.

Moga MM, Moore RY (1997) Organization of neural inputs to the suprachiasmatic nucleus in the rat. J Comp Neurol 389:508-534.

Moore RY (1996) Entrainment pathways and the functional organization of the circadian system. Prog Brain Res 111:103-119.

Moore RY, Card JP (1994) Intergeniculate leaflet: an anatomically and functionally distinct subdivision of the lateral geniculate complex. J Comp Neurol 344:403-430.

Moore RY, Speh JC (1993) GABA is the principal neurotransmitter of the circadian system. Neurosci Lett 150:112-116.

Morin LP (1994) The circadian visual system. Brain Res Rev 19:102-127.

Morin LP, Blanchard J (1995) Organization of the hamster intergeniculate leaflet: NPY and ENK projections to the suprachiasmatic nucleus, intergeniculate leaflet and posterior limitans nucleus. Vis Neurosci 12:57-67.

Morin LP, Blanchard JH (2001) Neuromodulator content of hamster intergeniculate leaflet neurons and their projection to the suprachiasmatic nucleus or visual midbrain. J Comp Neurol 437:79-90.

Morin LP, Blanchard JH (2005) Descending projections of the hamster intergeniculate leaflet: relationship to the sleep/arousal and visuomotor systems. J Comp Neurol 487:204-216.

Morinville A, Martin S, Lavallee M, Vincent JP, Beaudet A, Mazella J (2004) Internalization and trafficking of neurotensin via NTS3 receptors in HT29 cells. Int J Biochem Cell Biol 36:2153-2168.

Mrosovsky N (1996) Locomotor activity and non-photic influences on circadian clocks. Biol Rev Camb Philos Soc 71:343-372.

Obrietan K, van den Pol AN (1996) Neuropeptide Y depresses GABA-mediated calcium transients in developing suprachiasmatic nucleus neurons: a novel form of calcium long-term depression. J Neurosci 16:3521-3533.

Park HT, Baek SY, Kim BS, Kim JB, Kim JJ (1993) Profile of Fos-like immunoreactivity induction by light stimuli in the intergeniculate leaflet is different from that of the suprachiasmatic nucleus. Brain Res 610:334-339.

Paxinos G, Watson C (1998) The rat brain in stereotaxic coordinates. New York: Academic. 
Peyron C, Tighe DK, van den Pol AN, de Lecea L, Heller HC, Sutcliffe JG, Kilduff TS (1998) Neurons containing hypocretin (orexin) project to multiple neuronal systems. J Neurosci 18:9996-10015.

Pheng LH, Dumont Y, Fournier A, Chabot JG, Beaudet A, Quirion R (2003) Agonist- and antagonist-induced sequestration/internalization of neuropeptide $\mathrm{Y}$ Y1 receptors in HEK293 cells. Br J Pharmacol 139:695-704.

Pickard GE (1985) Bifurcating axons of retinal ganglion cells terminate in the hypothalamic suprachiasmatic nucleus and the intergeniculate leaflet of the thalamus. Neurosci Lett 55:211-217.

Pickard GE, Ralph MR, Menaker M (1987) The intergeniculate leaflet partially mediates effects of light on circadian rhythms. J Biol Rhythms 2:35-56.

Pinault D (1996) A novel single-cell staining procedure performed in vivo under electrophysiological control: morpho-functional features of juxtacellularly labeled thalamic cells and other central neurons with biocytin or Neurobiotin. J Neurosci Methods 65:113-136.

Rusak B, Zucker I (1979) Neural regulation of circadian rhythms. Physiol Rev 59:449-526.

Rusak B, Robertson HA, Wisden W, Hunt SP (1990) Light pulses that shift rhythms induce gene expression in the suprachiasmatic nucleus. Science 248:1237-1240.

Schaap J, Meijer JH (2001) Opposing effects of behavioural activity and light on neurons of the suprachiasmatic nucleus. Eur J Neurosci 13:1955-1962.

Shinohara K, Tominaga K, Fukuhara C, Otori Y, Inouye SI (1993) Processing of photic information within the intergeniculate leaflet of the lateral geniculate body: assessed by neuropeptide $\mathrm{Y}$ immunoreactivity in the suprachiasmatic nucleus of rats. Neuroscience 56:813-822.

Stornetta RL, Akey PJ, Guyenet PG (1999) Location and electrophysiological characterization of rostral medullary adrenergic neurons that contain neuropeptide $\mathrm{Y}$ mRNA in rat medulla. J Comp Neurol 415:482-500.

Takatsuji K, Tohyama M (1989) The organization of the rat lateral geniculate body by immunohistochemical analysis of neuroactive substances. Brain Res 480:198-209.

Tang IH, Murakami DM, Fuller CA (2002) Unilateral optic nerve transection alters light response of suprachiasmatic nucleus and intergeniculate leaflet. Am J Physiol Regul Integr Comp Physiol 282:R569-R577.

Vrang N, Mrosovsky N, Mikkelsen JD (2003) Afferent projections to the hamster intergeniculate leaflet demonstrated by retrograde and anterograde tracing. Brain Res Bull 59:267-288.

Weber ET, Rea MA (1997) Neuropeptide Y blocks light-induced phase advances but not delays of the circadian activity rhythm in hamsters. Neurosci Lett 231:159-162.

Zhang DX, Rusak B (1989) Photic sensitivity of geniculate neurons that project to the suprachiasmatic nuclei or the contralateral geniculate. Brain Res 504:161-164.

Zhao H, Rusak B (2005) Circadian firing-rate rhythms and light responses of rat habenular nucleus neurons in vivo and in vitro. Neuroscience 132:519-528.

\section{Neurobiotin, NPY and Orexin A}

\section{A. Confocal Image}

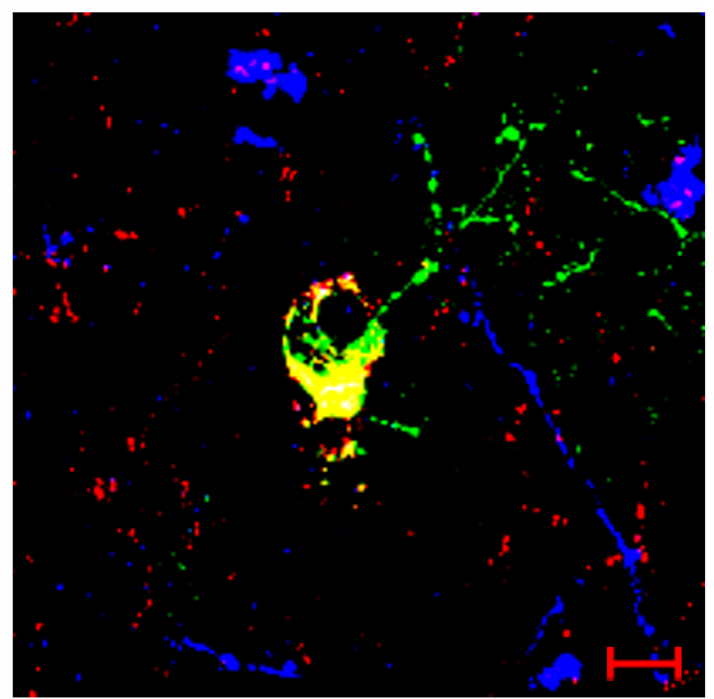

\section{Neurobiotin and NPY}

\section{B1. 3D \\ Reconstruction}

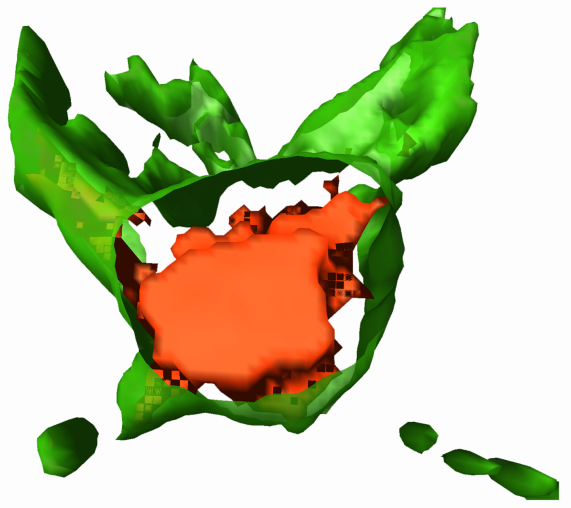

\section{Neurobiotin and Orexin A}

\section{B2. 3D \\ Reconstruction}

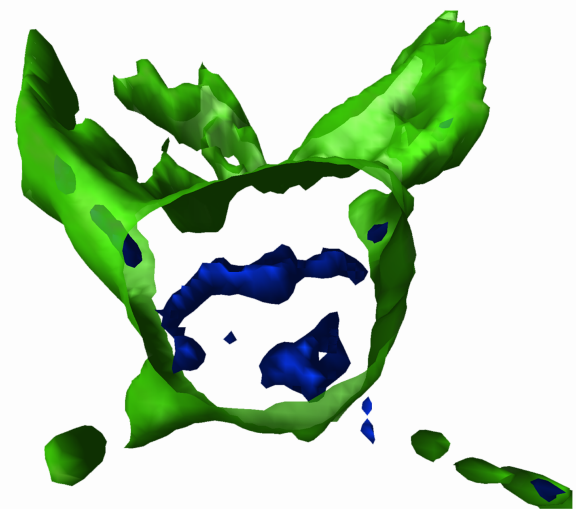

Figure 10. A, High-magnification confocal laser scanning fluorescence image of the soma of a VLG neuron that is considered NPY-containing. This neuron showed complex responses to retinal illumination. This merged image shows triple labeling for $\mathrm{Nb}$ (green), NPY (red), and orexin A (blue). Colocalization of Nb and NPY appears yellow, and colocalization of both with orexin A appears white. Scale bar, $20 \mu \mathrm{m}$. $\boldsymbol{B}$, Surface-rendered 3D reconstructions of the soma of this neuron from a stack of 11 optical sections at $1 \mu \mathrm{m}$ intervals. $\boldsymbol{B} 1$ shows the Nb-labeled soma and the extensive NPY content within the soma. $\boldsymbol{B} 2$ shows the $\mathrm{Nb}$-labeled soma and the small amounts of orexin $\mathrm{A}$ within the soma. 